\title{
Ecological influences on individual differences in color preference
}

\author{
Karen B. Schloss ${ }^{1}$ - Daniel Hawthorne-MadelI ${ }^{2}$ Stephen E. Palmer ${ }^{3}$
}

Published online: 14 August 2015

(C) The Psychonomic Society, Inc. 2015

\begin{abstract}
How can the large, systematic differences that exist between individuals' color preferences be explained? The ecological valence theory (Palmer \& Schloss, Proceedings of the National Academy of Sciences 107:8877-8882, 2010) posits that an individual's preference for each particular color is determined largely by his or her preferences for all correspondingly colored objects. Therefore, individuals should differ in their color preferences to the extent that they have different preferences for the same color-associated objects or that they experience different objects. Supporting this prediction, we found that individuals' color preferences were predicted better by their own preferences for correspondingly colored objects than by other peoples' preferences for the same objects. Moreover, the fit between color preferences and affect toward the colored objects was reliably improved when people's own idiosyncratic color-object associations were included in addition to a standard set of color-object associations. These and related results provide evidence that individual differences in color preferences are reliably influ-
\end{abstract}

Electronic supplementary material The online version of this article (doi:10.3758/s13414-015-0954-x) contains supplementary material, which is available to authorized users.

Karen B. Schloss

karenschloss@gmail.com

1 Department of Cognitive, Linguistic and Psychological Sciences, Brown University, Providence, RI, USA

2 Department of Psychology, Stanford University, Stanford, CA, USA

3 Department of Psychology, University of California, Berkeley, CA, USA enced by people's personal experiences with colored objects in their environment.

Keywords Color aesthetics · Color cognition · Ecological valence theory (EVT)

The study of human color preferences in psychology has an erratic history, largely due to the high degree of variability across individuals. Early researchers concluded that color preferences were too idiosyncratic for empirical study (e.g., Chandler, 1928; Cohn, 1884; von Allesch, 1924). However, Eysenck (1941) argued that these failures were due to the use of unstandardized colors and inadequate statistical analyses. His own results showed systematic effects with reliable agreement among participants, which were confirmed and extended by subsequent investigations of group averages (e.g., Granger, 1952, 1955; Guilford \& Smith, 1959; Hurlbert \& Ling, 2007; McManus, Jones, \& Cottrell, 1981; Ou, Luo, Woodcock, \& Wright, 2004; Palmer \& Schloss, 2010; Taylor \& Franklin, 2012). These studies indicated that average hue preferences follow a relatively smooth, curvilinear function, with a peak at blue and a trough around yellow to yellow-green. People generally prefer colors with greater saturation, although this result may be limited to abstract colored swatches (Schloss, Strauss, $\&$ Palmer, 2013). Color preferences may also increase with increased lightness (e.g., Guilford \& Smith, 1959; McManus et al., 1981), although this pattern is not always evident (e.g., Palmer \& Schloss, 2010). For reviews, see Whitfield and Wiltshire (1990) and Palmer, Schloss, and Sammartino (2013).

Despite robust, systematic patterns in average color preferences, there are still extensive individual differences. Indeed, the variability across individuals is large relative to the variability within individuals across testing sessions (McManus 
et al., 1981). How can these individual differences be explained?

\section{Biological accounts of individual differences}

One possibility is that individual differences are caused by differences in the responses of photoreceptors. Physiological differences in cone photopigments produce measureable differences in the threshold sensitivities to different colors, due to slight peak shifts in receptor response (e.g., the varieties of color weakness among the population of so-called normal trichromats). Even typical trichromats differ in their relative numbers of retinal L, M, and S cones (Roorda \& Williams, 1999), suggesting that such differences might underlie individual differences in color preferences. However, individual differences in the relative numbers of different cone types among normal trichromats have little effect on sensitive psychophysical measurements such as the locus of unique hues and color appearance ratings (e.g., Brainard et al., 2000; Webster, Miyahara, Malkoc, \& Raker, 2000). The visual system appears to have mechanisms that largely compensate for such differences, keeping color perception relatively invariant across the normal range of trichromatic retinal physiology. Unless color preferences are determined by perceptual attributes that are not consciously available, variations in lowlevel attributes are unlikely to be responsible for individual differences in color preferences among normal trichromats such as we study here.

Another biologically based account is Hurlbert and Ling's (2007) cone contrast model, which predicts color preferences based on the $L-M$ and $S-(L+M)$ cone contrasts in response to a color relative its background. This model was later extended to include brightness $(L+M+S)$ and a measure of saturation $\left(\mathrm{S}_{\mathrm{uv}}\right)^{1}$ (Ling \& Hurlbert, 2009). The extended model predicted $64 \%$ of the variance in the group averages of preferences for 90 colors with a weighted linear combination of the four predictors: $L-M, S-(L+M), L+M+S$, and $\mathrm{S}_{\mathrm{uv}}$.

\footnotetext{
${ }^{1} S, M$, and $L$ represent the outputs of the short-, medium-, and longwavelength-sensitive cones in the human retina: that is, the SmithPokorny cone fundamentals (Smith \& Pokorny, 1975). (See Eskew, McLellan, \& Giulianini, 1999, for the relevant formulation of cone contrasts, which are described in the supplementary materials of Hurlbert \& Ling, 2007.) The L-cone contrast value, $\Delta L$, was computed as $\left(L_{\mathrm{s}}-L_{\mathrm{b}}\right) / L_{\mathrm{b}}$; the M-cone contrast, $\Delta M$, was computed as $\left(M_{\mathrm{s}}-M_{\mathrm{b}}\right) / M_{\mathrm{b}}$; and the S-cone contrast was computed as $\Delta S=\left(S_{\mathrm{s}}-S_{\mathrm{b}}\right) / S_{\mathrm{b}}$, where the subscript "s" denotes the stimulus, and "b" the background color. The LM component cone contrast $L M_{\mathrm{c}}$ was computed as $L M_{\mathrm{c}}=0.7^{*} \Delta L-$ $0.72 * \Delta M+0.02 * \Delta S$, the S-component cone contrast $S_{\mathrm{c}}$ was computed as $0.8^{*} \Delta S-0.55^{*} \Delta L-0.25^{*} \Delta M$, and the luminance contrast $L u m_{\mathrm{c}}$ was computed as $0.9 * \Delta L+0.43^{*} \Delta M$ (Eskew et al., 1999). $S a t_{\mathrm{c}}$ represents the saturation coordinate of the colors in CIELUV space. The four-factor linear regression model that they reported was based on these four variables, as follows: $p_{\text {predict }}=w_{1} * S_{\mathrm{c}}+w_{2} * L M_{\mathrm{c}}+w_{3} * L u m_{\mathrm{c}}+w_{4} * S a t_{\mathrm{c}}+$ a.
}

Ling and Hurlbert used the same four-factor linear regression model to fit each observer's individual preferences for the same 90 colors, allowing the additive constant and the beta weights for each predictor to vary across individuals. This model explained an average of $48 \%$ of the variance in the individuals' color preference data. ${ }^{2}$ This approach provides a useful tool for predicting an individual's color preferences from a simple linear equation, once that individual's weights for the four predictors have been empirically established. However, it is unknown why individuals differ in the weights on their cone contrast and saturation dimensions. Hurlbert and Ling suggested that women weight more positively on the L$M$ axis, due to their early roles as gatherers in hunter-gatherer societies (e.g., needing to find red berries against green leaves). This sex difference in weightings on the L-M axis was not corroborated in other cultures, however (Taylor, Clifford, \& Franklin, 2013; Yokosawa, Schloss, Asano, \& Palmer, 2015) or in infants (Franklin, Bevis, Ling, \& Hurlbert, 2009), which challenges the gender-based evolutionary account for different weightings across individuals. How and why individual differences in color preferences arise, therefore, remain open questions.

A higher-level and purely psychophysical approach to understanding color preferences based on conscious color appearances is also possible, provided that preferences can be explained by how blue-versus-yellow, red-versus-green, lightversus-dark, and saturated-versus-desaturated the colors appear. Palmer and Schloss (2010) described and tested this model using a weighted average of the colors' rated values on these dimensions, roughly analogous to their coordinates in NCS color space (Hård \& Sivik, 1981). This model can also be used to describe individual differences in color preferences in a manner similar to the cone contrast model, using a fourfactor linear regression model to fit different observers. We note that such a model does not explain why different individuals have different weights on the four color appearance dimensions.

\section{An ecological account of individual differences}

Another approach to explaining color preferences is based on ecological factors: that is, differences in the ways that people respond to objects and cultural institutions in their environments. One such ecological account of color preferences has been formulated in the ecological valence theory (EVT; Palmer \& Schloss, 2010). The EVT posits that people like/ dislike a given color to the degree that they like/dislike all of

\footnotetext{
${ }^{2}$ For a smaller set of 24 colors, Hurlbert and Ling (2007) were able to predict up to $74 \%$ of the variance in average color preferences, and an average of $61 \%$ of the variance in individuals' color preferences. The model reported above, however, is more comprehensive, given its larger set of sampled colors.
} 
the objects and entities that they associate with that color. For example, people generally like saturated blues, in part, because they like clear sky and clean water, whereas they dislike browns, in part, because they dislike biological waste products and rotting food. This is not to say that people generally like all blue things or dislike all brown things, but only that their average preferences over all objects tend to be more positive for blue things and more negative for brown things. Supporting this approach, Palmer and Schloss found that $80 \%$ of the variance in average color preferences for 32 colors was explained by the average valences of all objects associated with the colors, weighted by how well the objects matched the associated colors (see further details below).

This ecological approach is based on the hypothesis that it will be adaptive for organisms to approach objects whose colors they like and to avoid objects whose colors they dislike, to the extent that their color preferences are correlated with objects that are beneficial versus harmful to them. Color preferences exist to perform an adaptive "steering" function, guiding behavior toward increased personal well-being. Although the original formulation of the EVT was couched in terms of biological adaptations for survival and reproduction of members of a species (Palmer \& Schloss, 2010), current evidence suggests that it is better formulated in terms of the result of learning by individuals about the conditions under which they believe they will thrive (i.e., to feel happy and prosperous) rather than fail (i.e., to feel sad and discontented). In making everyday decisions about how to behave (e.g., what to approach vs. avoid), individuals generally do so out of more nuanced concerns than species-based instincts for survival and reproduction. They are also influenced by more than just physical objects, including their affiliations with social, political, and cultural institutions (Schloss \& Palmer, 2014; Schloss, Poggesi, \& Palmer, 2011). The EVT does allow for color preferences to influence people's preferences for objects, especially when the same artifact is available in a variety of colors (e.g., clothes, cars, and furniture). However, the EVT implies that those color preferences originate from the valences of people's experiences with colored objects and that they will be reinforced via positive feedback to the extent that people like the things that they buy, make, or choose because of their color (Palmer \& Schloss, 2010).

We acknowledge that the EVT as currently formulated concerns preference for abstract colored patches, without reference to specific objects (e.g., the colors of clothes, furniture, or cars). Color preferences can and do vary for different kinds of objects (Holmes \& Buchanan, 1984; Saito, 1983; Schloss et al., 2013; Taft, 1997), and we refer the interested reader to Schloss et al. (2013) for a discussion of the relative contributions of the ecological, functional, and personal factors that account for preferences for the colors of particular objects.

The EVT predicts that individual differences in color preferences arise, at least in part, from differences in people's affective experiences with colored objects and with colorassociated entities in their physical, social, and cultural environments. There are two ways in which such effects might occur. One possibility is that different people can have different affective responses to the same objects and entities. If Nancy loves the taste of green vegetables and hates the taste of red berries, whereas Jim hates green vegetables and loves red berries, then, all else being equal, Nancy should like dark green colors more than Jim does, and Jim should like dark red more than Nancy does. The second possibility is that people have experiences with different objects. If Nancy loves playing cards with her grandmother on her grandmother's dark green rug, she will have a strongly positive affective response to this personally relevant object that Jim does not even know about. Likewise, Jim might have strongly positive associations with the bright red quilt that his wife gave him for his birthday soon after they began dating. According to the EVT, both Nancy's and Jim's different valences for the same objects and their experiences with different objects can cause differences in their relative color preferences for dark greens and bright reds. Such ecological accounts are not necessarily in conflict with the possibility of low-level physiological or psychophysical differences - both factors might be at workbut in this article we focus on examining the possible evidence for ecological effects.

The EVT approach implies that adult color preferences are not determined solely by hardwired mechanisms, but can change with experience. Evaluative conditioning provides a plausible mechanism underlying the formation and change of color preferences (De Houwer, Thomas, \& Baeyens, 2001; Hofmann, De Houwer, Perugini, Baeyens, \& Crombez, 2010) in that color preferences may be conditioned by the polarity and strength of the color's associations to a person's emotional evaluation of correspondingly colored objects. This learning account makes several predictions about developmental, social, and exposure-based differences in color preferences. We will briefly review the predictions and supporting evidence below.

Development of color preferences It is controversial whether color preferences are innate or learned (e.g., Adams, 1987; Bornstein, 1975). Early research on infant color preferences generally supported the innateness hypothesis, because young infants preferentially look most at blue and least at yellowgreen or yellow, roughly corresponding to explicit measures of adult preference (e.g., Adams, 1987; Bornstein, 1975; Franklin et al., 2008; Teller, Civan, \& Bronson-Castain, 2004; Zemach, Chang, \& Teller, 2007). However, most of these studies measured preferences for highly saturated colors, and a more recent study using less saturated, chroma-matched light and dark colors found distinct differences between 4- to 6-month-old infants and adults (Taylor, Schloss, Palmer, \& Franklin, 2013). Indeed, the correlation between infant and 
adult preferences was somewhat negative $(r=-.39, p>.05)$, where infants were biased to look toward dark yellow (the least preferred color for adults) and away from light blue (among the most preferred color for adults). To the extent that infant looking biases differ from adult color preferences, these results suggest that there must be developmental changes in color preferences. Although these changes could be due to maturational effects, this is unlikely, given the stability of color perception across the lifetime (Knoblauch, VitalDurand, \& Barbur, 2001; Petzold \& Sharpe, 1998; Roorda \& Williams, 1999). Absolute chromatic sensitivities vary over the lifespan (e.g., Banks \& Bennett, 1988), but psychophysical threshold measurements indicate that relative sensitivities along the three primary chromatic axes (protan, deutan, and tritan) change little, if at all, from the first 3-4 months of life to 86 years of age (Knoblauch et al., 2001). Other research on hue discrimination performance as a function of age found only slight differences between the ages of 4 and 25 years (Petzold \& Sharpe, 1998).

Differences due to social affiliations A learning-based version of the EVT predicts that people will learn to like colors that are strongly associated with sociocultural institutions that they like and to dislike colors that are strongly associated with sociocultural institutions that they dislike. Indeed, students at two rival universities (UC Berkeley and Stanford) liked their own school colors better than students at their rival school did, and the degree to which individuals preferred their own school's colors was correlated with their amount of selfreported school spirit (Schloss et al., 2011). Furthermore, a study comparing color preferences among US Republicans and Democrats for Republican red and Democrat blue found that the Republicans' and Democrats' color preferences changed to become more closely aligned with their own party's colors on Election Day in both 2010 and 2012, as compared to a non-Election-Day baseline (Schloss \& Palmer, 2014). Such changes in the color preferences of social groups over a few days are incompatible with genetic, physiological, or low-level psychophysical effects. These results support a learning-based account because, although it is logically possible that people may choose to attend particular universities or choose their political parties on the basis of preexisting color preferences, that seems unlikely.

Exposure-based differences An experimental manipulation showed that color preferences can also be modified by exposure to affectively biased samples of colored objects (Strauss, Schloss, \& Palmer, 2013). As compared to a pretest baseline, participants who experienced images of positive red objects (e.g., roses, strawberries) and negative green objects (e.g., vomit, snot) showed an increase in preferences for reds and a marginal decrease in preferences for greens, whereas others who saw positive green objects and negative red ones showed the opposite pattern. The degree to which individuals' color preferences changed during the experiment was predicted by the degree to which they liked the positive objects and disliked the negative objects. Given that these changes were observed within subjects within a single testing session, it is extremely unlikely that differences in color preferences can be explained by genetic and/or physiological differences.

\section{Testing the EVT for individual differences in color preferences}

In this article, we attempt to determine whether individual differences in color preferences can be explained in terms of ecological effects. Our approach is based on Palmer and Schloss's (2010) initial test of the EVT, but is modified for studying individual differences. The initial procedure assessed whether color preferences could be predicted by a measure of the extent to which people like/dislike the set of all objects that are associated with that color, called the weighted affective valence estimate (WAVE). The original WAVE calculation for each of 32 colors was based on the data produced by three separate groups of participants. One group viewed each of 32 colors and described the objects that they associated with each color. The second group rated the valence (positive/negative) of a condensed $\mathrm{set}^{3}$ of those objects. The third group rated how well the actual color of the objects matched the colors that elicited their description. The WAVE was computed for each color by multiplying the mean valence of each associated object by the mean match rating for that object-color pair (the weight), and then calculating the mean of those products. Formally, the WAVE for each color $\left(W_{\mathrm{c}}\right)$ is

$W_{c}=\frac{1}{n_{c}} \sum_{o=1}^{n_{c}} w_{c o} v_{o}$

where $c$ is the color, $w_{c o}$ is the average color-object match value for each pairing of color $c$ and object description $(o), v_{\mathrm{o}}$ is the average valence rating given to object $o$, and $n_{c}$ is the number of object descriptions ascribed to color $c$. The WAVEs for each of the 32 colors (Fig. 1d) explained $80 \%$ of the variance $(r=.893)$ in the color preferences of adults in the US (Fig. 1c), with no estimated free parameters. Taylor and Franklin (2012) replicated these findings using a subset of the colors in a group of British participants, for whom the WAVEs explained $66 \%$ of the variance $(r=.81)$.

Here, we studied individual differences by performing a modified and expanded version of the WAVE procedure using a within-subjects, repeated measures design. Each of 48 observers in the present experiment performed all three tasks required to calculate their own individual WAVE values for the 32 colors

\footnotetext{
${ }^{3}$ See Palmer and Schloss (2010) for the condensing procedure.
} 
A

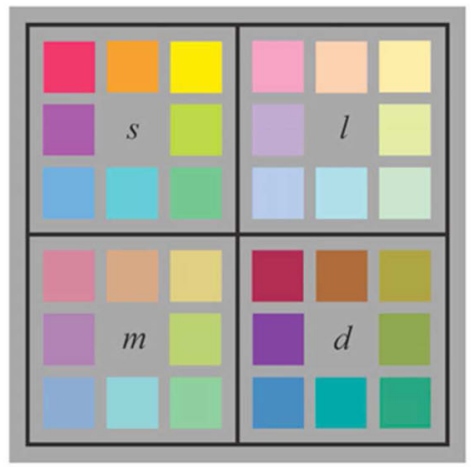

C

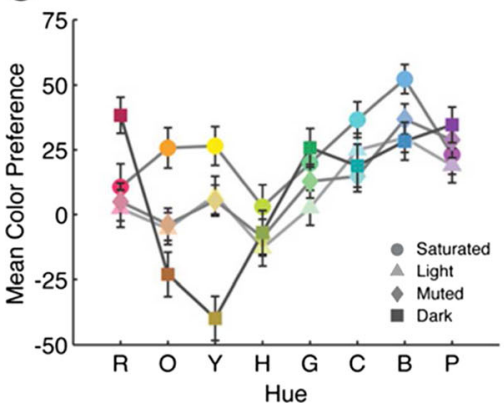

B

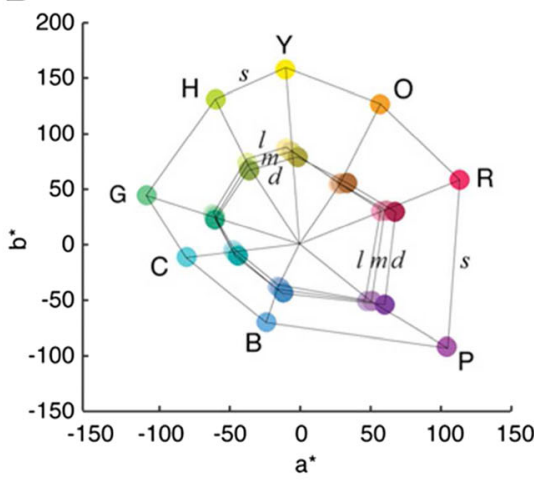

D

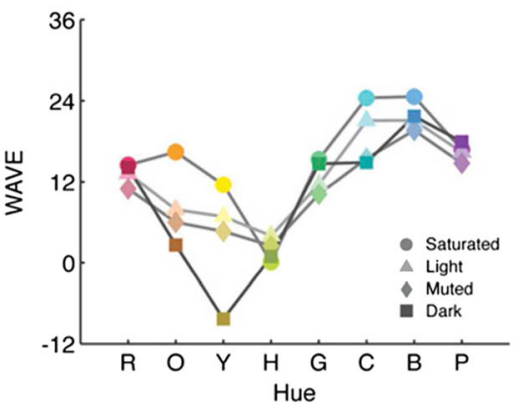

Fig. 1 (a) Approximations of the 32 Berkeley Color Project (BCP-32) chromatic colors used by Palmer and Schloss (2010), including eight hues at four lightness and saturation levels: saturated (s), light (l), muted (m), and dark (d). (b) Projections of the BCP-32 colors onto an isoluminant plane in CIELAB color space. (c) Average color preference ratings for the

BCP-32 colors (error bars represent standard errors of the means). (d) Weighted affective valence estimates (WAVEs) for the BCP-32 colors (which have no error bars because they represent data calculated from the group means of three different groups, as specified by Eq. 1). (From Palmer and Schloss 2010, Fig. 1)

studied. We tested the prediction that a given observer's personal WAVE measurements should be more strongly correlated with his or her own color preferences than with the color preferences of other observers: that is, that the within-subjects correlations of a person's WAVEs with his or her own color preferences should, on average, be higher than the between-subjects correlations of a person's WAVEs with other people's color preferences. The null hypothesis is that an individual's WAVE does not predict his or her color preferences any better than it predicts other individuals' preferences. The null hypothesis might fail to be rejected if WAVEs do capture a shared component of individuals' color preferences but cannot account for individual variability beyond this shared component.

We assessed these predictions using three different WAVE measures that differ only in the sets of objects that they include: personal WAVEs (P-WAVEs), idiosyncratic WAVEs (IWAVEs), and combined WAVEs (C-WAVEs). P-WAVEs are based on individual participants' valence ratings for the "standard" set of 222 object descriptions from Palmer and Schloss (2010). The P-WAVEs of different individuals will only differ if they have different valence ratings for the same 222 objects included in this set (see Table S1 in the supplementary materials for a complete list of these objects). This standard set

was designed to include objects only if they were believed to be known by all participants, so it leaves out all "idiosyncratic" objects that only one or a few individuals might know. IWAVEs are based on individual participants' valence ratings for the objects that they personally described as being associated with each color. Some of the objects could have been the same as those in the standard set (e.g., sun, leaves) if individuals reported them, but many were idiosyncratic objects that only the individual would know (e.g., "my favorite sweater" as an associate of a particular shade of blue). Comparisons of the relation between color preferences and P-WAVEs versus I-WAVEs should reveal the relative contributions of shared versus idiosyncratic object associations. C-WAVEs are computed for each subject on the basis of his/her valences of the standard objects plus those of his/her idiosyncratic objects, after eliminating redundancies for objects that were in both sets. The C-WAVEs thus provide the most complete test of the EVT's account of individual difference effects, because it contains WAVEs based on the largest sample of the objects relevant to each individual.

The present study also addresses two additional open questions about the EVT's ability to account for color preferences. The first question concerns whether the fit between color preferences and WAVEs previously reported by Palmer and 
Schloss (2010) was merely due to group-averaging effects. Strong positive correlations at the group mean level do not necessarily imply significant positive correlations at the individual level. Indeed, it is possible that group-average WAVEs would correlate with group-average color preferences even if people's own individual WAVEs (based on their own object valences) were unrelated to their own color preferences. To see how this is possible, consider two scenarios in which the correlations between the group-average color preferences and WAVEs are equally strong, yet the within-subjects correlations at the individual level vary. In the positive relation scenario, the EVT is true at both the individual and group levels: For every individual, the correlation between his/her own color preferences and WAVE scores is strong, in which case the average of the within-subjects WAVE-preference correlations would also be strong. In the no-relation scenario, imagine a different sample of individuals that give the same total set of WAVE scores and color preference ratings, but the pairings between the WAVE scores and the color preferences are randomized across individuals. This could result in no relation between WAVEs and color preferences at the individual level, in which case the average of the within-subjects WAVE-preference correlations would also be near zero. If the EVT can truly explain color preferences, then people's individual object valences should reliably predict some substantial portion of the variance in their individual color preferences, and not merely the variance at the group-average level.

The second question concerns whether the correlation between average color preferences and WAVEs in Palmer and Schloss (2010) was due to an emotional consistency bias in the objects that came to mind during the object description task. Potentially related biases toward emotionally congruent information have been documented in state-dependent memory effects: For instance, people are more likely to recall happy events when they are happy, and more likely to recall sad events when they are sad (Bower, 1981), although the opposite pattern has also been reported (Parrott \& Sabini, 1990). If analogous emotional consistency biases exist for accessing color-associated objects when viewing patches of color, people may have been more likely to describe positive objects while viewing preferable colors and negative objects while viewing disliked colors, independent of the true average valence of all objects of that color. If so, then the strong relation between color preferences and WAVEs could have been due to emotional consistency effects in the object association task. The analyses on the P-WAVEs in the present experiment are not open to this objection in accounting for individual differences in color preferences, because P-WAVEs are calculated from individuals' ratings of the same set of 222 standard objects. Although an emotional consistency bias could exist in this list of color-associated objects, that bias could not account for an individual's color preferences being predicted better by his/her own P-WAVEs than by other people's P-WAVEs.

\section{Method}

\section{Participants}

A total of 48 participants (mean age $=22$ years; 24 females, 24 males) completed all of the tasks in this study. All had normal color vision, as screened using the Dvorine Pseudoisochromatic Plates, and gave informed consent. The Committee for the Protection of Human Subjects at the University of California, Berkeley, approved the experimental protocol.

\section{Design, displays, and procedure}

Each participant completed four tasks, in the following order: color preference ratings, idiosyncratic object descriptions and object-color match ratings, standard object-color match ratings, and standard and idiosyncratic object valence ratings. Each task is described in a separate section below (see Fig. 2).

Color preference ratings This task was the same as that described in Palmer and Schloss (2010), except that each color preference was rated twice on one day and twice on another day to obtain more stable color preference data for each individual. Each color was presented as a small square $(100 \times 100$ pixels $)$ at the center of the screen of a 20 -in. iMac LCD computer monitor $(1,680 \times 1,050$ pixel resolution at $60-\mathrm{Hz}$ resolution) . The observer was positioned about $60 \mathrm{~cm}$ from the monitor in a dark room with no background illumination. The colors were the Berkeley Color Project (BCP) 32 set, which includes eight hues (red, orange, yellow, chartreuse, green, cyan, blue, and purple) at four saturation/lightness levels (saturated, light, muted, and dark); see Table 1 in the Appendix. The background color was a medium gray (CIE $x=.312, y=.318, Y=19.26$ ). The monitor was characterized using a Minolta CS100 Chroma Meter to ensure accurate color production.

Participants rated how much they liked each color on a 400-pixel line-mark rating scale by sliding the cursor to the appropriate location and clicking to record their response. The data were rescaled to range from -100 to +100 . Trials lasted until participants made their responses, and the next trial began $500 \mathrm{~ms}$ later. Before beginning the experimental trials, participants were given instructions to help them "anchor" the endpoints of the response scale. They were shown a display containing all 32 colors, asked to point to the color that they liked most, and were told that that color should be rated at or near the very much end of the line-mark scale. They were then asked to point to the color they liked least and were told that that color should be rated at or near the not at all end of the line-mark scale. The 32 colors were then presented individually in random order for ratings, as described above.

Idiosyncratic object descriptions and object-color match This task was completed on a different day, after all color 


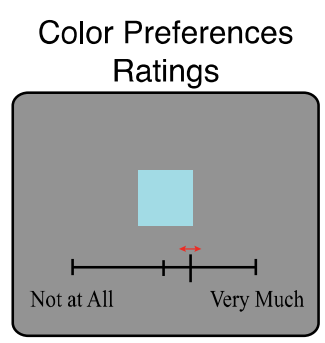

A

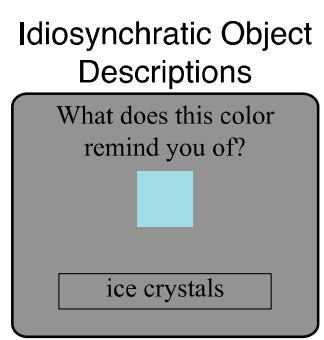

B

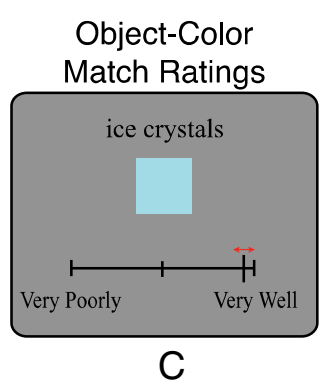

C

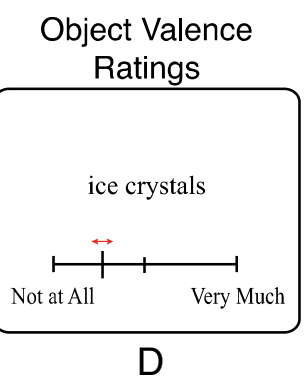

Fig. 2 Schematic representations of the stimulus screens for the four tasks, in order, of the within-subjects experimental procedure for measuring personal, idiosyncratic, and combined WAVEs (P-WAVEs, IWAVEs, and C-WAVEs, respectively). The same set of observers provided color preference ratings of the 32 colors (a), then provided

preference ratings were completed. Participants were presented with each of the 32 colors, one at a time, in random order and centered on the screen. They were instructed to think of as many objects as possible of which the color reminded them. They then typed a brief description of the object into a text box on the screen (e.g., "clear sky" or "rotten bananas") and pressed the Enter key. Next, they rated each object on how well the color on the screen matched the color of the object that they had just described, using a line-mark rating scale that ranged from very well to very poorly. After participants had described the object and provided an object-color match rating, the object name appeared on the left of the screen so that they could keep track of which objects they had already described. Once participants had finished describing objects for a particular color, they typed the word "done," and the next trial began with the next randomly selected color. Participants were told to describe any object that came to mind, even if it was "gross and disgusting." Participants were motivated to provide as many different object descriptions as they could by payments of $\$ 0.04$ for each unique object, $\$ 0.02$ for objects that were repeated once for other colors, and $\$ 0.01$ for objects that were repeated more than once. There was no time limit on completion of this task.

Standard object-color match ratings This task was the same as that described in Palmer and Schloss (2010) and was completed in a subsequent experimental session. For each color, participants were presented with each of the standard objects, along with the color with which it was described, and they rated how well the color of the described object matched the color on the screen. They rated all of the objects for a particular color before going on to the next color. Both the color order and object order were randomized for each color. Prior to starting the trials for each color, participants were shown a list of all the objects for that color so they would know what matching well and matching poorly meant in the context of the object set.

Object valence ratings (both standard and idiosyncratic) Object valence ratings were completed in the next and final

descriptions of as many objects as they could think of that were associated with the color presented (b), then made ratings of the match (degree of similarity) between the object and the color with which it was associated (c), and finally rated how much they liked each object (d)

experimental session. The first part of this task was the same as the object valence rating task described in Palmer and Schloss (2010). Participants were presented with each of the standard 222 object descriptions, displayed as black text on a white background, one at a time in random order. Their task was to make a line-mark rating of how much they liked each object on a scale from not at all to very much. Each object remained on the screen until participants made their response, and the next trial began $500 \mathrm{~ms}$ later. Prior to beginning, participants were shown a representative sample of objects so that they could anchor what not at all and very much meant for them in the context of the object set. The second part of this task was the same as the first part, but participants rated the valences of the idiosyncratic objects that they had described earlier in the object description task. There were no other differences between the standard and idiosyncratic sets of trials.

\section{Results and discussion}

\section{Reliability of color preference judgments}

We first examined the stability of individuals' color preferences within each testing session. Preference judgments in the first and second blocks were strongly correlated within Session 1 (average $r=.78)^{4}$ and within Session 2 (average $r=.84$ ). A paired-samples $t$ test comparing individuals' correlations between Session 1 and Session 2 indicated that they were significantly stronger in Session

\footnotetext{
${ }^{4}$ Before averaging the correlations, we computed their hyperbolic arctangent to decompress their lower and upper limits. We then transformed the average back to a traditional correlation space $(-1$ to +1$)$ by computing their hyperbolic tangent. All of the subsequent averaging and statistical tests of correlations reported in this article were conducted on such transformed correlations, and the average correlations reported have been transformed back by computing their hyperbolic tangents. These transformations have minimal effects on mid-range correlations, and have larger effects as the correlations approach \pm 1 . The majority of the correlations reported here were mid-range and were therefore minimally affected.
} 

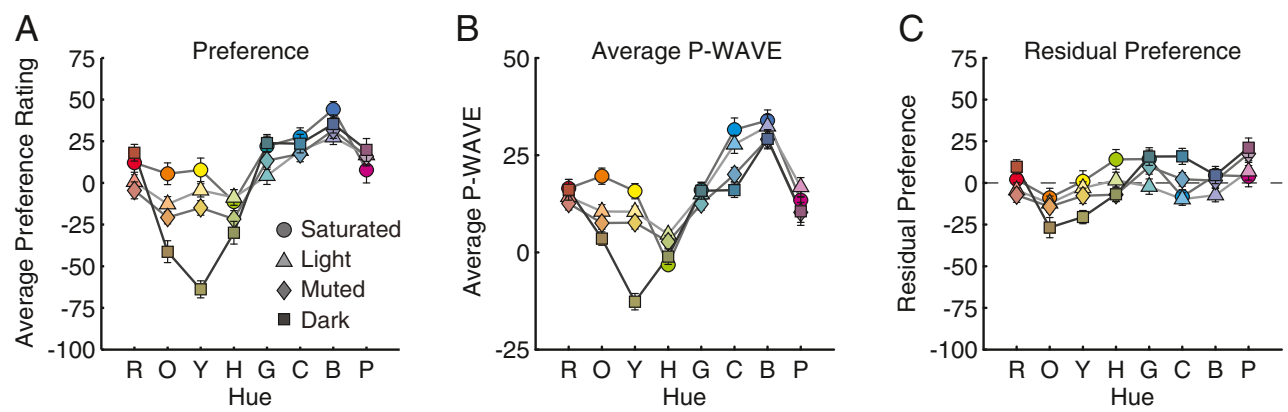

Fig. 3 (a) Average color preference ratings, (b) P-WAVE values, and (c) the residual differences between panels A and B after best-fit rescaling of the PWAVEs. (See the text for further explanations.) Error bars represent standard errors of the means

$2[t(47)=4.90, p<.001]$, suggesting that preference judgments stabilized over time. The correlation between individuals' color preferences (averaged over testing blocks) between Sessions 1 and 2 was also strong (average $r=.85$ ), indicating that color preferences were consistent across testing sessions.

We next tested whether there was more variability in color preferences between individuals than within individuals, as had previously been found by McManus et al. (1981). We computed a measure of between-subjects variability by correlating each individual's color preferences (averaged over the four testing blocks) with every other individual's color preferences and averaging over those correlations for each participant (average $r=.32$ ). We compared this measure of between-subjects variability to two measures of within-subjects variability: (1) the correlation between each individual's Block 1 and Block 2 ratings within Session 1 (average $r=.78$ ) and (2) the correlation between each individual's Session 1 and Session 2 ratings, averaged over the testing blocks within each session (average $r=.85$ ). Paired-samples $t$ tests indicated that the betweensubjects correlations were significantly weaker than the within-subjects correlations within Session $1[t(47)=20.45, p$ $<.001]$ and than the within-subjects correlations over testing sessions $[t(47)=25.41, p<.001]$. These results strongly support the previous findings of McManus et al. (1981).

We also compared the present participants' average color preference ratings (Fig. 3a) with the color preferences reported for a different sample of 48 participants from the same Northern California population (Fig. 1c; Palmer \& Schloss, 2010). These two data sets were nearly identical $(r=.97)$. A three-way analysis of variance (ANOVA; 8 hues $\times 4$ cuts $\times 2$ groups) revealed no interactions between group and hue $[F(7$, $658)=1.47, p>.05]$, group and cut $(F<1)$, or group, hue, and cut $[F(21,1974)=1.17, p>.05]$.

\section{Within-subjects replication of prior WAVE results}

The WAVEs reported by Palmer and Schloss (2010) (Fig. 1d) were calculated using the between-subjects data sets (i.e., different people producing the object-color associations, object valence, and object-color match judgments). We compared these group-average, betweensubjects WAVEs to the average within-subjects PWAVEs (Fig. 3b), which were calculated from individuals' object-valence ratings and object-color match ratings of the standard set of 222 objects. The original WAVE and the present mean P-WAVE measurements were very highly correlated $(r=.94)[t(30)=15.09, p<$ $.001] .^{5}$ (See Table S1 in the supplementary materials for a complete list of the 222 standard objects and their average valence ratings and standard deviations in the present experiment.) The correlation between the present participants' group-average color preferences with their groupaverage P-WAVEs was $.87[t(30)=9.67, p<.001]$, which is comparable to the corresponding correlation of .89 that Palmer and Schloss reported using the same set of 222 object descriptions. Therefore, the within-subjects PWAVE procedure gave essentially the same results as those obtained in the between-subjects WAVE procedure when testing corresponding correlations between groupaveraged color preferences and WAVEs.

\section{Accounting for individual differences with P-WAVEs}

Does the correlation between color preferences and WAVEs observed in the group averages described by Palmer and Schloss (2010) and replicated here also hold at the individual level? To address this question, we first computed the correlation between each individual's preferences for the 32 colors with his or her P-WAVE for each color (subsequently referred to as $W S-P)$. The mean WS-P over the 48 participants $(r=.55)$ was significantly greater than zero $[t(47)=16.02, p<.001)$ (see Fig. 4). P-WAVEs thus predicted color preferences at the individual level, and the strong correlations between the

\footnotetext{
${ }_{5}^{5}$ ANOVAs cannot be used to compare these two data sets, because there are no individual scores for the between-subjects WAVEs from Palmer and Schloss (2010). Their WAVEs were calculated by averaging the data from three different groups performing the three component tasks of the WAVE procedure, and thus can only be treated as 32 values with no error variance.
} 


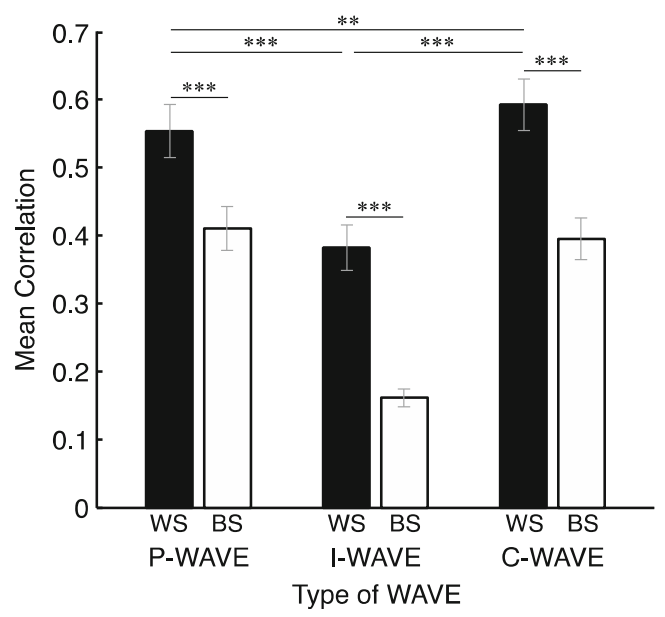

Fig. 4 Means of the individual participants' within-subjects (WS; black bars) and between-subjects (BS; white bars) correlations between color preferences and P-WAVEs, I-WAVEs, and C-WAVEs. Error bars represent standard errors of the means. ${ }^{* *} p<.01,{ }^{* * *} p<.001$

group-average color preferences and WAVEs reported above and in Palmer and Schloss's original study are not merely due to averaging effects. We note that it is expected that the mean WS-P (.55) should be lower than the correlation between the mean color preferences and mean P-WAVEs reported above (.87), because data at the individual level are inherently noisier than data at the group mean level.

Next, we tested the EVT's central prediction about individual differences: An individual's color preferences should be predicted better by his or her own P-WAVEs than by other participants' P-WAVEs. To address this question, we first correlated each participant's color preferences with every other participant's P-WAVEs and then calculated the mean of these between-subjects correlations for each participant (subsequently referred to as $B S-P$ ). We then conducted a pairedsamples $t$ test to determine whether the mean WS-P (average $r=.55$ ) was greater than the mean BS-P (average $r=.41$ ) (Fig. 4). Supporting the EVT's basic prediction, the mean WS$\mathrm{P}$ was significantly greater than the mean BS-P $[t(47)=7.87, p$ $<.001]$. Furthermore, the WS-P was greater than the BS-P for 41 of the 48 participants $\left[\chi^{2}(1)=24.08, p<.001\right] .{ }^{6}$ At least some portion of the individual differences in color preferences is therefore linked to corresponding individual differences in

\footnotetext{
${ }^{6}$ It is not surprising that the mean BS-P (.41) would be lower than the corresponding correlation between the between-subjects correlation between group-average color preferences and WAVEs in Palmer and Schloss (2010) (.89), because the correlations between individual participants' data should be noisier than the correlations between average data. In addition, other effects for individuals might not be captured by the PWAVEs as presently defined, including (at least) their idiosyncratic object valences and experiences, their symbolic and/or conceptual associations to colors, their typical visual environments, and their beliefs about the compatibility of different colors with their hair, eye, and skin colors. Many of these effects presumably cancelled out when measured across participants in the group-average data from Palmer and Schloss, but did not cancel out in the individual data presented here.
}

their preferences for the ecological objects associated with those colors.

A related prediction of the EVT is that any partition of the 48 individuals into $n$ groups of people with similar color preferences (as defined by higher average within-group similarity in color preferences than average between-group similarity) should exhibit stronger within-group color-preference/PWAVE correlations than between-group color-preference/PWAVE correlations. To test this prediction, we conducted a $k$ means clustering analysis (Maechler, Rousseeuw, Struyf, Hubert, \& Hornik, 2002) for each value of $k$ from two clusters to 48 clusters (i.e., the 48 individual participants), based solely on the pairwise correlations between individuals' 32 color preferences. (Importantly, the P-WAVE data were not considered in creating these clusters.) We then calculated the correlations between preferences and P-WAVEs for each participant, and then averaged the within-cluster correlations and betweencluster correlations. Figure 5 (solid lines) shows the average within-cluster and between-cluster correlations for a subset of the values of $k^{7}$ Consistent with the EVT, the within-cluster correlations are systematically higher than the between-cluster correlations $[F(1,47)=227.72, p<.001]$. The predictive value of the within-cluster P-WAVE increased as the number of clusters increased to the individual level $(k=48, r=.55)$, with a significant linear contrast $[F(1,47)=19.23, p<.001]$. This positive slope indicates that, as clusters became more individualized, the correspondingly more individualized WAVEs were better predictors of color preferences. In comparison, the average between-cluster correlations remained constant, around .40 for all values of $k$ (linear contrast $F<1$ ).

We also performed the corresponding analyses using $k$-means to cluster individuals according to their P-WAVE data (rather than their color preferences, as described above). The dashed lines in Fig. 5 show the average within-group and between-group correlations for each value of $k$. Again, the within-cluster correlations are higher overall for the sampled values of $k[F(1,47)=157.64$, $p<.001]$. A repeated measures ANOVA comparing the withinsubjects correlations for the two clustering methods (clustering by color preference vs. by P-WAVEs) and six levels of $k(2,4,8,16$, and 32) revealed no effect of clustering method $[F(1,47)=1.69$, $p>.05]$ and no interaction with $k(F<1){ }^{8}$

We also conducted a more extensive analysis of the $k=2$ clustering solution based on similarities of color preferences, and found that the differences between the average preferences of the two clusters of individuals were characterized well by how natural versus synthetic the colors were judged to be (see Fig. S1 and the supplementary materials for details). A similar analysis conducted separately for males and females

\footnotetext{
${ }^{7}$ To attenuate correlations between nearby clustering solutions, $k$ was sampled at each power of 2 below the total number of participants (i.e., $2,4,8,16$, and 32 ), plus the full 48 subjects.

${ }^{8}$ The values for $k=48$ were omitted because they are necessarily the same in both clustering solutions.
} 


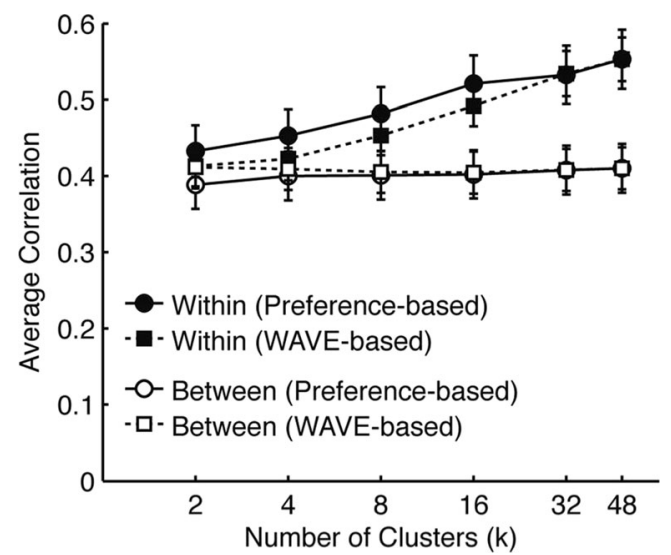

Fig. 5 Averages of the within- and between-cluster correlations between color preferences and P-WAVEs, when the data are $k$-means clustered by color preferences (circles) and $k$-means clustered by P-WAVEs (squares). Error bars represent standard errors of the means

and a discussion of gender differences can also be found in the supplementary materials (Fig. S2).

\section{Idiosyncratic color-object associations and I-WAVEs}

We next tested whether the within-subjects I-WAVEs (WS-I) were more strongly correlated with individuals' color preferences than were the between-subjects I-WAVEs (BS-I), and compared the WS-I correlations to the WS-P correlations described above. Recall that I-WAVEs differ from P-WAVEs in that they are calculated from the valence and color-match ratings of only the idiosyncratic object descriptions provided by the individual participants - including objects whose color others would not know, such as "my favorite sweater"-rather than those of the 222 standard object descriptions. They also differ in that we did not require the objects listed in the I-WAVE sets to be concrete physical objects, allowing conceptual and/or symbolic associations, such as "happy" for yellows and "violence" for reds. The average correlation of each individual's color preferences and IWAVEs (WS-I) was .38, which was significantly greater than zero $[t(47)=12.01, p<.001]$ and greater than the mean BS-I [average $r=.16 ; t(47)=7.26, p<.001$ ] (Fig. 4). However, the mean WS-I (.38) was significantly weaker than the mean WS-P (.55) described above $[t(47)=5.47, p<.001]$.

Why should the highly individualized I-WAVEs predict individuals' color preferences less well than the more general PWAVEs? One possibility is that individuals do not generate all (or even very many) of the objects that are associated with a given color in a single sitting. Although individuals might not think of a particular object for a particular color during the task (e.g., "twilight sky" for dark blue), they still might agree that such an object is a good associate for that color, and their color preferences might be influenced by how much they like many of the objects they did not generate themselves. The standard set of objects that underlie P-WAVEs were generated from the aggregate of 74 participants' object descriptions (Palmer \& Schloss,
2010) and contain significantly more objects per color (an average of 27.03) than do the idiosyncratic sets of objects that underlie I-WAVEs (an average of 4.27) $[t(31)=14.00, p<.001]$. Therefore, the P-WAVEs are based on far more complete information about an individual's weighted object valences, even though the objects on which it is based are less personalized.

The degree to which the I-WAVEs were related to color preferences varied substantially across participants, with WS-I ranging from -.23 to +.75 . If WAVEs based on more objects (i.e., containing more complete information) explain more variance in color preferences, then it is possible that the differences between individuals in their I-WAVE correlations may be due in part to the number of objects that individuals produced in the object association task. The "law of large numbers"- that is, that the average obtained from a large number of observations should be closer to the expected value as the number of observations increases-implies that the more objects a person produces in his or her idiosyncratic color-object association task, the better the resulting IWAVEs would estimate his or her preferences for the set of all objects associated with those colors. Indeed, the number of objects that a person described in the object association task (averaged over colors) was positively correlated $(r=.38)$ $[t(46)=2.79, p=.008]$ with how well his/her I-WAVEs predicted his/her color preferences. To determine whether this finding might have been due to some more general relation between WAVEs and particular people's color preferences, we conducted the same analysis, but used the correlations between their color preferences and their P-WAVEs (rather than their I-WAVEs), which should not depend on the number of idiosyncratic objects produced. This correlation was unreliably negative $(r=-.10)$, which supports the possibility that the predictive power of I-WAVEs for color preference depends on the number of objects on which it is based.

Taylor and Franklin (2012) reported that, on average, participants preferred colors that were associated with fewer objects: $r$ $=-.76$ in their British data and $r=-.66$ in Palmer and Schloss's (2010) US data. The reason for this negative relation was unclear, but Taylor and Franklin suggested that it could be due to fluency (i.e., colors with fewer associates are easier to process and are thereby preferred) and/or to attention (i.e., disliked colors draw more attention and thereby elicit more object associations within the allotted time). To examine whether this negative correlation was present at the individual level, we correlated each participant's color preferences with the number of objects that he or she reported for each color and conducted a $t$ test on these correlations to determine whether this distribution was different from zero. ${ }^{9}$ We found no evidence that the average correlation was negative (average $r=.06$ ) [t(47) $=1.88, p>.05]$. Therefore, whatever factors might cause an association

\footnotetext{
${ }^{9}$ One participant reported the same number of objects for each color, producing an undefined correlation, which we coded as zero (no relation).
} 
between fewer objects and more preferred colors at the level of group averages do not appear to operate at the level of individual participants, at least when the latter are given unlimited time to report color-object associations.

\section{Combined color-object associations and C-WAVEs}

The P-WAVEs alone are superior to the highly individualized I-WAVEs alone in predicting individual differences in color preferences, but do the idiosyncratic objects in the I-WAVE explain any additional variance beyond that captured by the PWAVE? To address this question, we generated a "combined" WAVE (or C-WAVE) by merging the standard and idiosyncratic object lists for each participant, as follows. If there were exact duplicates on the two lists (e.g. "sun" on both the standard and idiosyncratic lists), we averaged the valence and object-color match ratings for each color with which it was associated. If two objects were judged to be conceptually similar but were not identical (e.g., "sun" and "sunlight"), we treated them as one object and averaged the data, provided that the valence ratings were within \pm 10 units $(5 \%)$ on the 200-unit response scale. However, if the conceptually similar objects had valence ratings that differed by more than \pm 10 units, they were treated as separate objects.

Although the difference was not large, the correlation between the individuals' C-WAVEs and color preferences (WS-C, average $r=.59$ ) was greater than the correlation between their P-WAVEs and color preferences (WS-P, average $r=.55$ ) [t(47) $=3.29, p<.01]$ (Fig. 4). Moreover, this was true for 39 out of the 48 participants $\left[\chi^{2}(1)=18.75, p<.001\right]$. The mean WS-C was also reliably greater than the mean of the between-subject correlations of C-WAVEs and color preferences (BSC, average $r=.39)[t(47)=9.14, p<.001]$. These results indicate that people's idiosyncratic object valences contribute additional information not available in the standard objects in the P-WAVE. It is also possible that the difference might be due, in part, to the fact that the C-WAVE includes more objects than the P-WAVE.

\section{Serial output order of object associations and emotional bias}

We mentioned in the introduction that the strong correlation between color preferences and WAVEs reported by Palmer and Schloss (2010) might be due to an emotional consistency bias operating in the object association task. That is, the sets of objects associated with the colors obtained in the WAVE procedure would be emotionally biased by affective consistency if people tended to produce liked objects for liked colors and disliked objects for disliked colors during the object association task. Such an emotional consistency bias in the set of objects associated with colors might then cause spuriously high correlations between our measured WAVEs and color preferences when compared with the correlation based on the full and unbiased set of relevant objects. (We note that it is unclear how this full set of objects could be compiled in practice, given that the object descriptions are often not single words or standard phrases that would appear in a dictionary: e.g., "clear sky," "rotten bananas," and "dead foliage").

One heuristic process that would produce such a bias in a time-limited output series would be that people generate objects they like earlier in their output lists for colors they like and generate objects they dislike earlier in their output lists for colors they dislike. Truncating the list before all relevant objects had been described would then produce the hypothesized affective bias in the sample of objects, which would not be present in the complete set of objects. If that occurred, it would inflate the correlation between WAVEs and color preferences.

We examined whether the output order of objects was emotionally biased (i.e., whether the valence of earlier objects was consistent with the valence of their color) by correlating the serial output order and object valences for each participant, contingent on the valence of the object (see the supplementary materials for details). The results showed that the average correlation was only weakly positive $(r=.10)$, and the mean of the distribution of individuals' correlations was not significantly different from zero $[t(47)=1.97, p>.05]$. The results therefore provide no evidence for an emotional consistency bias in the object descriptions, at least in the early portion of an individual's association list (see the supplementary materials for further results and discussion).

\section{Cone contrast and color appearance models}

In a final set of analyses, we evaluated how well the cone contrast and color appearance models account for the individual differences in color preferences reported here, and we compared their predictive performance with that of the C-WAVEs. To evaluate Ling and Hurlbert's (2009) four-factor cone contrast model $\left[L-M, S-(L+M), L+M, S_{\mathrm{uv}}\right]$, we conducted a multiple linear regression to predict each participant's color preferences, allowing the weights on each predictor to vary across participants. The resulting models explained an average of $39 \%$ of the variance, which was not reliably more than the $37 \%$ explained by the C-WAVEs ${ }^{10}[t(47)<1]$. However, the cone contrast model has four predictors per person, and the $\mathrm{C}$ WAVE model has only one predictor, so there is far more opportunity for curve fitting in the cone contrast model. It is also unclear why different participants would have different weights on the cone contrasts, but the EVT's C-WAVEs provide an explanatory account for why individuals' color preferences vary. We note, however, that computing C-WAVE is far more methodologically cumbersome than fitting the cone

\footnotetext{
${ }^{10}$ This value $\left(R^{2}=.37\right)$ is the average of the $R^{2}$ values for each individual, which is algebraically different from the square of the average of the individual's correlations reported earlier $\left(r=.59 ; R^{2}=.35\right)$. We used this average $R^{2}$ value because it was comparable to the average $R^{2}$ value for the four-factor cone contrast model.
} 
contrast model. Therefore, if the goal of subsequent research is to describe individuals' color preferences patterns with a linear equation, the cone contrast model will be more efficient.

To test whether the C-WAVE predictor explains variance independent from that explained by the cone contrast model predictors, we conducted a regression model for each participant using all five predictors. This new hybrid model explained an average of $61 \%$ of the variance in individuals' color preferences, substantially more than the $39 \%$ explained by the cone contrast model alone, with only one additional parameter. This result suggests that cone contrasts and WAVEs capture independent aspects of color preferences.

We also fit Palmer and Schloss's (2010) color appearance model (based on participant ratings of red-green, yellowblue, light-dark, and saturated-desaturated; see Table S2 in the supplementary materials) to the individual data reported above. This model explained an average of $53 \%$ of the variance. A paired-samples $t$ test comparing the $R^{2} \mathrm{~s}$ for individuals using the color appearance model versus the cone contrast model indicated that the color appearance model provided better fits $[t(47)=7.05, p<.001]$. Both models have four predictors per participant, and are therefore directly comparable. The difference between them is roughly similar to the one from Palmer and Schloss's results fitting these two models to group-average color preferences, where the cone contrast model explained $34 \%$ of the variance and the color appearance model explained $60 \%$. The color appearance model also explained significantly more variance $(53 \%)$ than the CWAVEs (37\%) at the level of individual observers, as indicated by a paired-samples $t$ test comparing the individuals' $R^{2}$ values for the two models [t(47) $=4.13, p<.001]$, but at the expense of requiring three additional predictor variables per person. A model containing both the C-WAVE and color appearance predictors explained $65 \%$ of the variance, which was better than the $53 \%$ explained by the color appearance model alone. However, as we explained for the cone contrast model, its additional parameters allowed for additional curve fitting ${ }^{11}$ and is theoretically less informative. The color

\footnotetext{
${ }^{11}$ As was reported by Palmer and Schloss (2010), the WAVE model explained more variance in their group-average data $(80 \%)$ than did the cone contrast model (37\%) and the color appearance model (59\%). With the present individuals' data, the C-WAVE model did less well, both in absolute terms ( $37 \%$ vs. the previous $80 \%$ ) and relative to the cone contrast ( $37 \%$ vs. $39 \%)$ and color appearance ( $37 \%$ vs. $53 \%)$ models. The absolute difference was due to trying to account for the much larger and noisier data set: 1,536 data points from individuals ( 32 colors for each of 48 participants) versus 32 data points ( 32 colors averaged over individuals) with no free parameters. The difference relative to the other two models is probably due to the larger disparity in the numbers of free parameters. In the group-average data, the WAVE model has three fewer predictors than the other two models, whereas in the present individual data, the C-WAVE model has 144 fewer parameters than the other two models. The extra degrees of freedom provide a huge benefit for the other two models in fitting the much larger individual data set that is unavailable to the C-WAVE.
}

appearance model provides a description of individual differences with no obvious explanation for why they occur, whereas the EVT-based C-WAVE model provides a causal explanation of the individual differences for which it accounts: That is, they are due to differences in people's valences for the objects associated with the corresponding colors.

Finally, a regression model including all three sets of predictors accounted for a total of $73 \%$ of the variance, on average, but we note that it included nine predictor variables per participant to predict 32 color preferences. These additional parameters might simply increase the curve-fitting power of the model without adding further explanatory power.

\section{General discussion}

The goal of this research was evaluate whether the EVT can account for individual differences in color preferences. Specifically, we tested the prediction that individuals should differ systematically in their color preferences in ways that reflect their experiences with different objects and/or their different affective responses to the same objects. The present results using within-subjects measurements of the WAVEs for the same 32 colors studied by Palmer and Schloss (2010) showed that people's color preferences were predicted better by their own preferences for correspondingly colored objects (WS-P) than by other people's preferences for the same objects (BS-P). The results also showed that the combined WAVEs (C-WAVEs) of individuals' preferences for the objects that they personally associate with the colors (I-WAVEs) and their preferences for the standard objects (P-WAVEs) explain their color preferences better than their preferences for the standard objects do alone. These results support the EVT's claim that people's personal color preferences are systematically shaped by their own preferences for correspondingly colored objects. We also extended previous tests of the EVT (Palmer \& Schloss, 2010) using a different set of 48 participants and a within-subjects version of the WAVE procedure. The results replicated the strong correlation between groupaverage color preferences and WAVEs.

In addition, we compared the EVT's WAVE measures with predictions derived from the augmented cone contrast model (Ling \& Hurlbert, 2009), which has previously been used to account for individual differences in color preferences. This model is based on the difference between the responses of S, M, and $L$ retinal cones to a color relative to its background color. Their extended model $\left[L-M, S-(L+M), L+M+S, S_{\mathrm{uv}}\right]$ explained an average of $39 \%$ of the variance in the present data from individuals, in which the weighting on each factor was estimated for each individual. This four-predictor model fit the data no better than the one-predictor C-WAVE model, which explained $37 \%$ of the variance. Although the cone contrast model provides an elegant description of individual differences in color preferences in 
terms of relatively low-level mechanisms, it is unclear from this approach why individuals would differ in their weightings.

The C-WAVE explains an average of $37 \%$ of the variance in individuals' color preferences, which is far less than the original WAVE measurements explained of the variance in groupaverage preferences $(80 \%)$. It is to be expected that fits to individual participants will be weaker than fits to group averages, because there is more noise in individuals' data. However, with only $37 \%$ of the variance explained, additional factors must contribute to color preferences. Might additional variables help explain variance from an ecological perspective?

One factor that we are currently exploring is the role of abstract symbolism in people's color preferences. We briefly mentioned one aspect of this issue in discussing the differences between the color preferences of people in Cluster 1 versus Cluster 2 (see Fig. S1) as being potentially being related to differential preferences for natural versus synthetic colors. For example, do people who like nature a great deal tend to prefer "natural" colors more than people who do not? Although abstract symbols were permitted in the I-WAVEs, they were actively omitted from the standard set of objects in the P-WAVE, as described by Palmer and Schloss (2010). P-WAVEs are more comprehensive than IWAVEs because the object descriptions are pooled across participants. Therefore, to evaluate whether symbolic associations account for additional variability in individual differences, it will be necessary to repeat the original WAVE procedure, but for symbolic associations rather than object associations. We are currently testing the influences of symbolic and conceptual factors on color preferences in China and the US, and preliminary evidence suggests that symbolic factors are much more important in China.

Another factor is one's emotional response to the visual environment in which one is living. Do people who like living in the American Southwest tend to like the warm desaturated colors so prevalent in their environment (e.g., pale yellows, browns, oranges, and reds), and do those who dislike living there tend to dislike those same colors? Do color preferences change systematically over the seasons in regions of the world where different color palettes are associated with different seasons? Do people prefer colors that they believe "go well with" their personal hair and eye color? These and other potentially relevant factors should be addressed in future research to understand the nature of ecological influences on the substantial differences in color preferences that are so evident between different individuals.

\footnotetext{
Author note We thank Kelly Whiteford, Lily Lin, and Eli Strauss for their considerable efforts in helping to compile and analyze aspects of this large data set. We thank Mike Webster for useful consultations about psychophysical and physiological issues in color perception, and Noah Goodman and Joseph Austerweil for assistance with the analyses. We thank Rosa Poggesi and Thomas Langlois for coordinating the testing sessions, and many undergraduate volunteers for helping to run the participants. Finally, we thank Jeremy Wolfe, Stephen Westland, Valerie Bonnardel, and an anonymous reviewer for their extensive comments and critiques on previous drafts of this article. The research reported here
}

was supported by Grant Numbers BCS-0745820 and BCS-1059088 from NSF to S.E.P. and by a Google gift to S.E.P.

\section{Appendix}

Table 1 CIE 1931 values and Munsell values for the 32 chromatic colors (Palmer \& Schloss, 2010)

\begin{tabular}{|c|c|c|c|c|c|c|}
\hline Color & & $x$ & $y$ & $Y$ & Hue & Value/Chroma \\
\hline \multirow[t]{4}{*}{ Red } & Saturated & .549 & .313 & 22.93 & $5 \mathrm{R}$ & $5 / 15$ \\
\hline & Light & .407 & .326 & 49.95 & $5 \mathrm{R}$ & $7 / 8$ \\
\hline & Muted & .441 & .324 & 22.93 & $5 \mathrm{R}$ & $5 / 8$ \\
\hline & Dark & .506 & .311 & 7.60 & $5 \mathrm{R}$ & $3 / 8$ \\
\hline \multirow[t]{4}{*}{ Orange } & Saturated & .513 & .412 & 49.95 & $5 \mathrm{YR}$ & $7 / 13$ \\
\hline & Light & .399 & .366 & 68.56 & $5 \mathrm{YR}$ & $8 / 6$ \\
\hline & Muted & .423 & .375 & 34.86 & $5 \mathrm{YR}$ & $6 / 6$ \\
\hline & Dark & .481 & .388 & 10.76 & $5 \mathrm{YR}$ & $3.5 / 6$ \\
\hline \multirow[t]{4}{*}{ Yellow } & Saturated & .446 & .472 & 91.25 & $5 \mathrm{Y}$ & $9 / 12$ \\
\hline & Light & .391 & .413 & 91.25 & $5 \mathrm{Y}$ & $9 / 6.5$ \\
\hline & Muted & .407 & .426 & 49.95 & $5 \mathrm{Y}$ & $7 / 6.5$ \\
\hline & Dark & .437 & .450 & 18.43 & $5 \mathrm{Y}$ & $5 / 6.5$ \\
\hline \multirow[t]{4}{*}{ Chartreuse } & Saturated & .387 & .504 & 68.56 & $5 \mathrm{GY}$ & $8 / 11$ \\
\hline & Light & .357 & .420 & 79.90 & $5 \mathrm{GY}$ & $8.5 / 6$ \\
\hline & Muted & .360 & .436 & 42.40 & $5 \mathrm{GY}$ & $6.5 / 6$ \\
\hline & Dark & .369 & .473 & 18.43 & $5 \mathrm{GY}$ & $4.5 / 6$ \\
\hline \multirow[t]{4}{*}{ Green } & Saturated & .254 & .449 & 42.40 & $3.75 \mathrm{G}$ & $6.5 / 11.5$ \\
\hline & Light & .288 & .381 & 63.90 & $3.75 \mathrm{G}$ & $7.75 / 6.25$ \\
\hline & Muted & .281 & .392 & 34.86 & $3.75 \mathrm{G}$ & $6 / 6.25$ \\
\hline & Dark & .261 & .419 & 12.34 & $3.75 \mathrm{G}$ & $3.75 / 6.25$ \\
\hline \multirow[t]{4}{*}{ Cyan } & Saturated & .226 & .335 & 49.95 & $5 \mathrm{BG}$ & $7 / 9$ \\
\hline & Light & .267 & .330 & 68.56 & $5 \mathrm{BG}$ & $8 / 5$ \\
\hline & Muted & .254 & .328 & 34.86 & $5 \mathrm{BG}$ & $6 / 5$ \\
\hline & Dark & .233 & .324 & 13.92 & $5 \mathrm{BG}$ & $4 / 5$ \\
\hline \multirow[t]{4}{*}{ Blue } & Saturated & .200 & .230 & 34.86 & $10 \mathrm{~B}$ & $6 / 10$ \\
\hline & Light & .255 & .278 & 59.25 & $10 \mathrm{~B}$ & $7.5 / 5.5$ \\
\hline & Muted & .241 & .265 & 28.90 & $10 \mathrm{~B}$ & $5.5 / 5.5$ \\
\hline & Dark & .212 & .236 & 10.76 & $10 \mathrm{~B}$ & $3.5 / 5.5$ \\
\hline \multirow[t]{4}{*}{ Purple } & Saturated & .272 & .156 & 18.43 & $5 \mathrm{P}$ & $4.5 / 17$ \\
\hline & Light & .290 & .242 & 49.95 & $5 \mathrm{P}$ & $7 / 9$ \\
\hline & Muted & .287 & .222 & 22.93 & $5 \mathrm{P}$ & $5 / 9$ \\
\hline & Dark & .280 & .181 & 7.60 & $5 \mathrm{P}$ & $3 / 9$ \\
\hline
\end{tabular}

\section{References}

Adams, R. J. (1987). An evaluation of color preference in early infancy. Infant Behavior \& Development, 10, 143-150.

Banks, M. S., \& Bennett, P. J. (1988). Optical and photoreceptor immaturities limit the spatial and chromatic vision of human neonates. Journal of the Optical Society of America A, 5, 2059-2079. doi: 10.1364/JOSAA.5.002059 
Bornstein, M. H. (1975). Qualities of color vision in infancy. Journal of Experimental Child Psychology, 19, 401-419.

Bower, G. H. (1981). Mood and memory. American Psychologist, 36, 129-148. doi:10.1037/0003-066X.36.2.129

Brainard, D., Roorda, A., Yamauchi, Y., Calderone, J., Metha, A., Neitz, M., \& Jacobs, G. (2000). Functional consequences of the relative numbers of L and M cones. Journal of the Optical Society of America A, 17, 607-614.

Chandler, A. R. (1928). Recent experiments on visual aesthetics. Psychological Bulletin, 25, 720-732. doi:10.1037/h0074055

Cohn, J. (1884). Experimentelle Untersuchungen tiber die Gefuhlsbetonung der Farben, Helligkeiten und ihre Combinationen. Philosophische Studien, 10, 562-602.

De Houwer, J., Thomas, S., \& Baeyens, F. (2001). Association learning of likes and dislikes: A review of 25 years of research on human evaluative conditioning. Psychological Bulletin, 127, 853-869. doi:10. 1037/0033-2909.127.6.853

Eskew, R. T., McLellan, J. S., \& Giulianini, F. (1999). Chromatic detection and discrimination Color vision: From genes to perception (pp. 345-368). Cambridge, UK: Cambridge University Press.

Eysenck, H. J. (1941). A critical and experimental study of color preference. American Journal of Psychology, 54, 385-391.

Franklin, A., Bevis, L., Ling, Y., \& Hurlbert, A. (2009). Biological components of colour preference in infancy. Developmental Science, 13, 346-354. doi:10.1111/j.1467-7687.2009.00884.x

Franklin, A., Pitchford, N., Hart, L., Davies, I. R. L., Clausse, S., \& Jennings, S. (2008). Salience of primary and secondary colours in infancy. British Journal of Developmental Psychology, 26, 471483.

Granger, G. W. (1952). Objectivity of colour preferences. Nature, 170, $178-180$.

Granger, G. W. (1955). An experimental study of colour preferences. Journal of General Psychology, 52, 3-20.

Guilford, J. P., \& Smith, P. C. (1959). A system of color-preferences. American Journal of Psychology, 72, 487-502.

Hård, A., \& Sivik, L. (1981). NCS—Natural Color System: A Swedish standard for coloer notation. Color Research and Application, 6, 129-138.

Hofmann, W., De Houwer, J., Perugini, M., Baeyens, F., \& Crombez, G. (2010). Evaluative conditioning in humans: A meta-analysis. Psychological Bulletin, 136, 390-421. doi:10.1037/a0018916

Holmes, C. B., \& Buchanan, J. A. (1984). Color preference as a function of the object described. Bulletin of the Psychonomic Society, 22, 423-425.

Hurlbert, A. C., \& Ling, Y. (2007). Biological components of sex differences in color preference. Current Biology, 17, R623-R625. doi:10. 1016/j.cub.2007.06.022

Knoblauch, K., Vital-Durand, F., \& Barbur, J. L. (2001). Variation of chromatic sensitivity across the life span. Vision Research, 41, 2336.

Ling, Y. L., \& Hurlbert, A. C. (2009, November). A new model for color preference: Universality and individuality. Paper presented at the 15th Color Imaging Conference, Arlington, VA.

Maechler, M., Rousseeuw, P., Struyf, A., Hubert, M., \& Hornik, K. (2002). cluster: Cluster analysis basics and extensions (R package version 1.14.2). Retrieved from http://cran.r-project.org/web/ packages/cluster/index.html

McManus, I. C., Jones, A. L., \& Cottrell, J. (1981). The aesthetics of colour. Perception, 10, 651-666.

Ou, L.-C., Luo, M. R., Woodcock, A., \& Wright, A. (2004). A study of colour emotion and colour preference. Part III: Colour preference modeling. Color Research and Application, 29, 381-389.
Palmer, S. E., \& Schloss, K. B. (2010). An ecological valence theory of human color preference. Proceedings of the National Academy of Sciences, 107, 8877-8882. doi:10.1073/pnas.0906172107

Palmer, S. E., Schloss, K. B., \& Sammartino, J. (2013). Visual aesthetics and human preference. Annual Review of Psychology, 64, 77-107. doi:10.1146/annurev-psych-120710-100504

Parrott, W. G., \& Sabini, J. (1990). Mood and memory under natural conditions: Evidence for mood incongruent recall. Journal of Personality and Social Psychology, 59, 321-336.

Petzold, A., \& Sharpe, L. T. (1998). Hue memory and discrimination in young children. Vision Research, 38, 3759-3772.

Roorda, A., \& Williams, D. R. (1999). The arrangement of the three cone classes in the living human eye. Nature, 397, 520-522.

Saito, T. (1983). Latent spaces of color preference with and without a context: Using the shape of an automobile as the context. Color Research and Application, 8, 101-113.

Schloss, K. B., \& Palmer, S. E. (2014). The politics of color: Preferences for Republican red versus Democratic blue. Psychonomic Bulletin \& Review, 21, 1481-1488. doi:10.3758/s13423-014-0635-0

Schloss, K. B., Poggesi, R. M., \& Palmer, S. E. (2011). Effects of university affiliation and "school spirit" on color preferences: Berkeley versus Stanford. Psychonomic Bulletin \& Review, 18, 498-504. doi: 10.3758/s13423-011-0073-1

Schloss, K. B., Strauss, E. D., \& Palmer, S. E. (2013). Object color preferences. Color Research and Application, 38, 393-411.

Smith, V. C., \& Pokorny, J. (1975). Spectral sensitivity of the foveal cone photopigments between 400 and $500 \mathrm{~nm}$. Vision Research, 15, 161171.

Strauss, E. D., Schloss, K. B., \& Palmer, S. E. (2013). Color preferences change after experience with liked/disliked colored objects. Psychonomic Bulletin \& Review, 20, 935-943. doi:10.3758/ s13423-013-0423-2

Taft, C. (1997). Color meaning and context: Comparisons of semantic ratings of colors on samples and objects. Color Research and Application, 22, 40-50.

Taylor, C., Clifford, A., \& Franklin, A. (2013a). Color preferences are not universal. Journal of Experimental Psychology: General, 142, 1015-1027. doi:10.1037/a0030273

Taylor, C., \& Franklin, A. (2012). The relationship between color-object associations and color preference: Further investigation of ecological valence theory. Psychonomic Bulletin \& Review, 19, 190-197. doi:10.3758/s13423-012-0222-1

Taylor, C., Schloss, K., Palmer, S. E., \& Franklin, A. (2013b). Color preferences in infants and adults are different. Psychonomic Bulletin \& Review, 20, 916-922. doi:10.3758/s13423-013-0411-6

Teller, D. Y., Civan, A., \& Bronson-Castain, K. (2004). Infants' spontaneous color preferences are not due to adult-like brightness variations. Visual Neuroscience, 21, 397-402.

von Allesch, G. J. (1924). Die aesthetische Erscheinungsweise der Farben. Psychologische Forschung, 6, 1-91.

Webster, M. A., Miyahara, E., Malkoc, G., \& Raker, V. E. (2000). Variations in normal color vision: II. Unique hues. Journal of the Optical Society of America A, 17, 1545-1555. doi:10.1364/JOSAA. 17.001545

Whitfield, T., \& Wiltshire, T. (1990). Color psychology: A critical review. Genetic, Social, and General Psychology Monographs, 116, 387411.

Yokosawa, K., Schloss, K. B., Asano, M., \& Palmer, S. E. (2015). Crosscultural studies of color preferences: US and Japan. Cognitive Science, in press.

Zemach, I., Chang, S., \& Teller, D. Y. (2007). Infant color vision: Prediction of infants' spontaneous color preferences. Vision Research, 47, 1368-1381. doi:10.1016/j.visres.2006.09.024 